\title{
Novel fiber Bragg grating sensor implemented in a polymer-core/silica- cladding hybrid optical fiber
}

Zhijun Yan, Hani Kbashi, Kaiming Zhou, Lin Zhang

Zhijun Yan, Hani Kbashi, Kaiming Zhou, Lin Zhang, "Novel fiber Bragg grating sensor implemented in a polymer-core/silica-cladding hybrid optical fiber," Proc. SPIE 9157, 23rd International Conference on Optical Fibre Sensors, 91578C (2 June 2014); doi: 10.1117/12.2059560

EDIE Event: OFS2014 23rd International Conference on Optical Fiber Sensors, 2014, Santander, Spain 


\title{
Novel Fiber Bragg grating sensor implemented in a polymer- core/silica-cladding hybrid optical fiber
}

\author{
Zhijun Yan, Hani Kbashi, Kaiming Zhou*, Lin Zhang \\ Aston Institute of Photonic Technologies, Aston University, Birmingham, B4 7ET, United Kingdom
}

\begin{abstract}
A polymer-core/silica-cladding hybrid optical fiber is implemented by filling a capillary with UV-curable epoxy and a following UV-laser scanning exposure. A fiber Bragg grating is successfully inscribed in parallel using a phase mask. The experimental results show a reduced thermal response for the FBG and a theoretical analysis for such a hybrid optical fiber is performed which corroborates existing of a turning temperature for minimized thermal response.
\end{abstract}

Keywords: fiber Bragg grating, hybrid optical fiber, polymer

\section{INTRODUCTION}

As an outstanding optical sensor, fiber Bragg grating (FBG) has been used to monitor a range of physical parameters including temperature, strain, loading, pressure and etc [1]. While seemingly omnipotent, for most sensing applications except temperature, FBGs have to remove their thermal cross-talking, for which several configurations have been proposed: a dual grating with one for sensing and the other as a temperature reference [2], external components exerting strain or bending to the grating to balance the thermal induced wavelength shift [3]. These schemes are complicated, increasing device size, limiting their application and with higher cost. Temperature compensation of long period grating (LPG) was suggested with proper design of index profile of the fiber or co-doping of material of negative thermo optic coefficient (TOC)[4,5]. For FBG, Kim proposed a cladding-removed optical fiber submerged in a liquid of negative thermo-optical TOC and demonstrated low thermal response [6]. However, the cladding-removed fiber is very delicate which limits its application for sensing. K Zhou implemented a hybrid optical waveguide using a polymer of negative TOC in a micro-channel which is nevertheless delicate too [7]. Here we describe implementation of a hybrid optical fiber composed of a silica-cladding and polymer-core and inscription of a FBG with a phase mask. With analysis of its thermal response, we demonstrate a low thermal response can be obtained.

\section{THEORETICAL ANALYSIS}

The effective refractive index (RI) of the fundamental mode of an optical fiber, a key factor determining the Bragg wavelength, is dependent on the materials and geometry constructing the fiber, i.e. RI of materials and radius of the core. Most standard optical fiber built on silica have a TOC of $\sim 10^{-5}$ which gives rise to a $\sim 0.01 \mathrm{~nm} /{ }^{\circ} \mathrm{C}$ thermal response for FBGs of $1550 \mathrm{~nm}$ if the thermal expansion effect is neglected. However, if the fiber consists of materials with TOC of opposite signs, there would be conditions where its FBG would show minimal thermal response due to the cancelling effect of the two materials. Most polymer has negative TOC with values ranging from $1 \times 10^{-4}$ to $4 \times 10^{-4}$ [8] and most of all, they offer excellent optical properties with low transmission loss.

Thermal induced variation to the optical property of the fiber, i.e. its effective RI, can be modelled by solving the standard characteristic function of the EM field. However, for single mode fiber, there exist an empirical expression for calculation of the normalized propagation constant and thus the effective index [9]:

$$
b=(1.1428-0.996 / V)^{2} \text { for } 1.5<V<2.5
$$

where $V=\frac{2 \pi \cdot a}{\lambda} \sqrt{n_{\text {core }}^{2}-n_{\text {clad }}^{2}}$ is the normalized frequency and $n_{\text {core }}$ and $n_{\text {clad }}$ are RI of the core and cladding of the fiber respectively and are dependent on temperature. 
The relation between $b$ and effective RI of fiber is thus given by

$$
n_{\text {eff }}=\sqrt{n_{\text {clad }}^{2}+b\left(n_{\text {core }}^{2}-n_{\text {clad }}^{2}\right)}
$$

Figure 1 gives the variation of the effective RI with respect to the temperature. With proper design of the fiber, a turning point with a minimal thermal response can be seen in the figure around room temperature $\left(25^{\circ} \mathrm{C}\right)$. The parameter used for the simulation are :

\begin{tabular}{|l|l|l|l|}
\hline$n_{\text {core }}$ & 1.451 & $\begin{array}{l}\text { TOC } \\
\text { cladding }\left(1 /{ }^{\circ} \mathrm{C}\right)\end{array}$ & $1.2 \times 10^{-5}$ \\
\hline$n_{\text {clad }}$ & 1.444 & Diameter of core & $5 \mu \mathrm{m}$ \\
\hline $\begin{array}{l}\text { TOC of } \\
\text { core }\left(1 /{ }^{\circ} \mathrm{C}\right)\end{array}$ & $1 \times 10^{-4}$ & Wavelength & $1.55 \mu \mathrm{m}$ \\
\hline
\end{tabular}

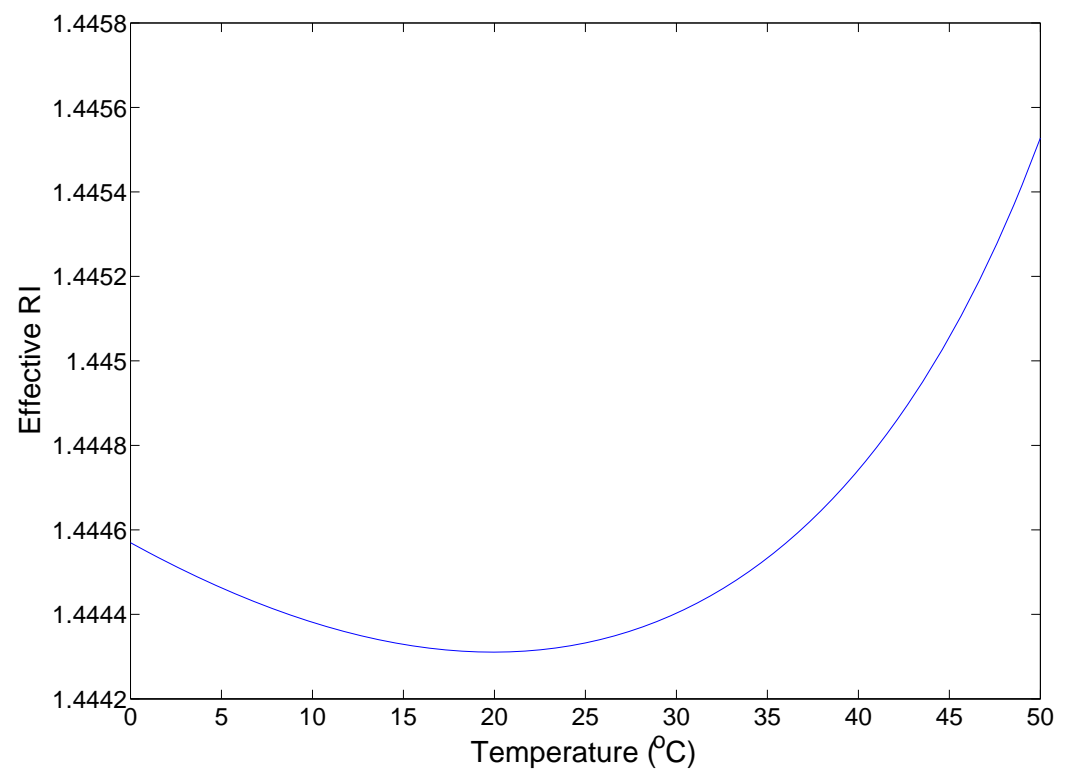

Figure 1 Temperature induced variation to effective RI of the hybrid polymer/glass optical fiber. Around room temperature a minimal thermal response is demonstrated.

\section{EXPERIMENT}

The first step to fabricate the hybrid optical fiber is to inject UV-curable adhesive into a micro-capillary made of fuse silica. The adhesive (NOA89 from Norland) is of low viscosity to facilitate its infusion into the capillary and a syringe pump is used. Its RI is larger than that of silica so that light can be confined in the core region to form a waveguide. Compatible with a standard silica optical fiber, the capillary has an inner-diameter of $5 \mu \mathrm{m}$ and an outer diameter of $125 \mu \mathrm{m}$ for the cladding. Figure 2 shows the image of the capillary before (a) and after (b) filling of UV adhesive and the difference is apparent. 


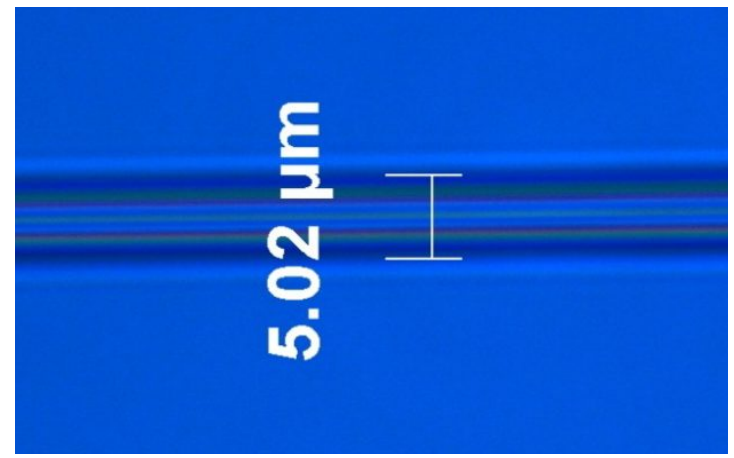

(a)

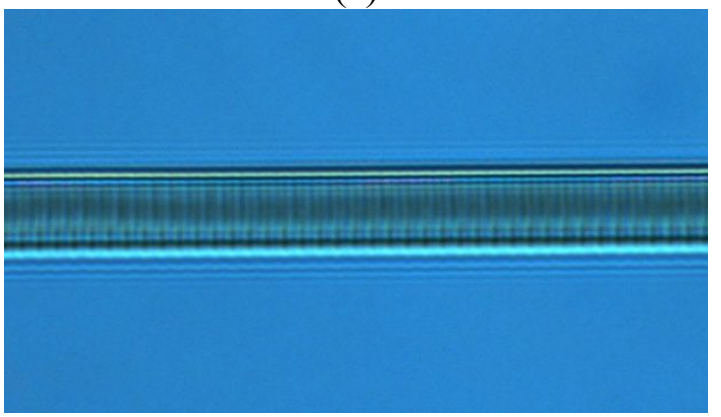

(c)

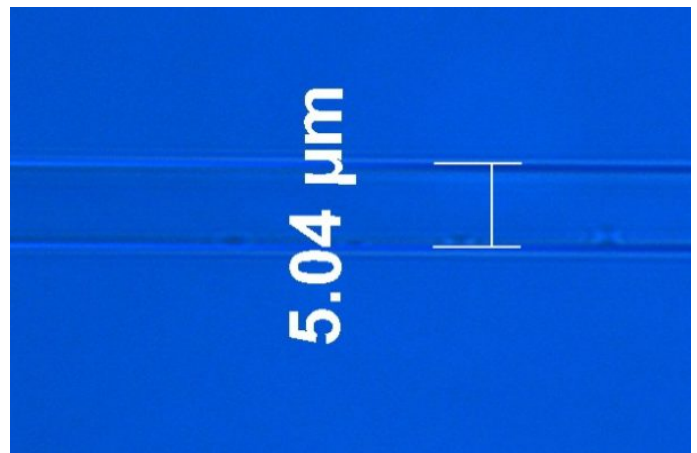

(b)

Figure 2 the micro-image of hollow core filled with air (a) and UV adhesive (b); (c) grating structure taken under a $\times 100$ objective lens.

The second step is to cure the adhesive. We use a scanning UV laser beam instead of a lamp with an intention to inscribe a FBG in parallel. A $10 \mathrm{~cm}$ fiber with freshly filled adhesive is kept straight and illuminated with a scanning $244 \mathrm{~nm} \mathrm{Ar+}$ ion UV laser beam. During the exposure, part of the fiber is placed behind a phase mask of a period of $1058 \mathrm{~nm}$. Because the adhesive has very high photosensitivity, during grating inscription, we adopt a very lower laser power density $\left(15 \mathrm{~mW}, \sim 2 \mathrm{~W} / \mathrm{mm}^{2}\right)$ and faster scanning speed $(0.2 \mathrm{~mm} / \mathrm{sec})$. Figure $2 \mathrm{c}$ gives the image of the grating showing the periodic structure clearly. For characterization, a mechanic splicing (from Thorlab) is used to couple light from and to standard optical fiber. Index matching gel is applied at the ends of the fiber to eliminate the Fresnel reflection. The reflection spectrum of the FBG is shown in Figure 3 (a) and a distinct Bragg peak at $1532 \mathrm{~nm}$ is demonstrated. Though the specs for NO89 gives a RI of 1.51, it is for visible light and RI for near infrared has not been measured. Bragg wavelength $\left(\lambda_{B}\right)$ is used to calculate the effective index $n_{\text {eff }}$ with $\lambda_{B}=2 \cdot n_{\text {eff }} \cdot \Lambda$ and then RI for the cured polymer is found to be 1.455 . V value of the fiber at this wavelength is 1.83 which implies it is a single mode.

The thermal response of the FBG was examined by heating the sample at different temperature and the reflection spectra are shown in Figure 3(b). This initial experiment shows the Bragg peak of the grating remain unchanged for $20^{\circ} \mathrm{C}$ and $40^{\circ} \mathrm{C}$, though further heating decrease the Bragg wavelength due to the negative TOC of the polymer. 

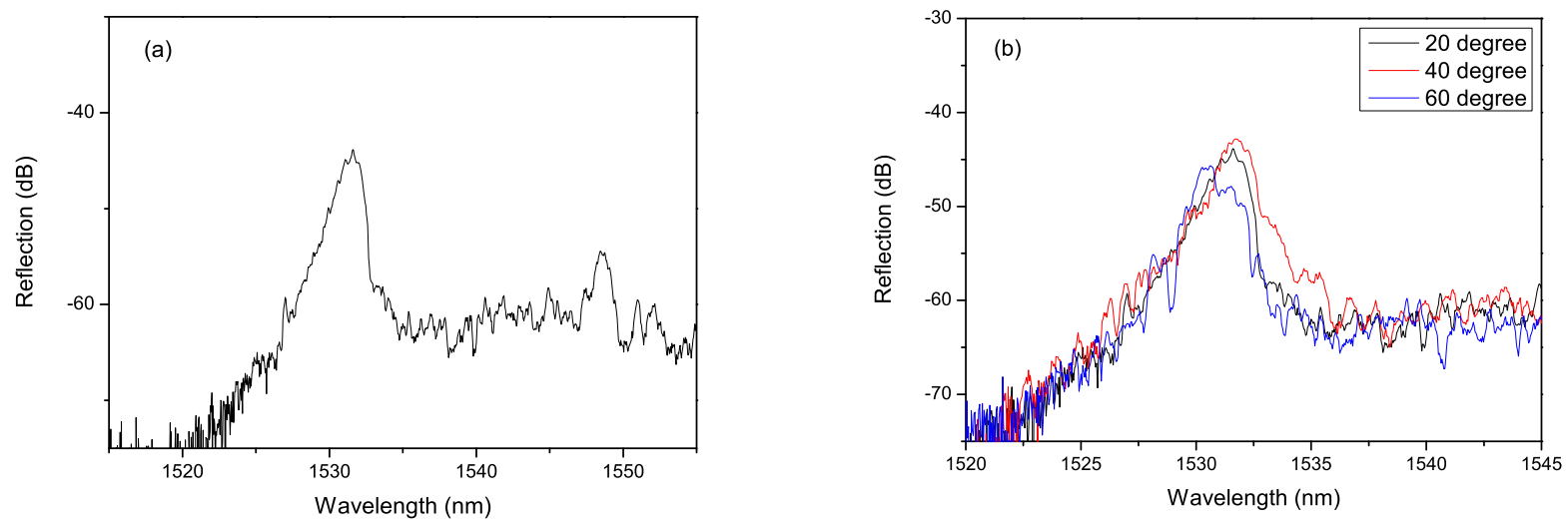

Figure 2 the reflection spectra of FBG in polymer core based fiber (a) at room temperature and (b) at different temperature.

\section{CONCLUSION}

We demonstrate a process for fabrication of a polymer-core/silica-cladding hybrid optical fiber which shows single mode operation at $\sim 1550 \mathrm{~nm}$. A FBG is inscribed in parallel and thermal characterization show low thermal sensitivity at room temperature. Theoretical analysis of the fiber is carried out which predict a turning temperature of such minimal thermal response.

\section{REFERENCES}

[1] Raman Kashyap, [Fiber Bragg grating], Academic Press, 441-496, (2009)

[2] Dong, X.Y.; Liu, Y.; Liu, Z.; Dong, X.Y. "Simulations displacement and temperature measurement with cantilever-based fiber Bragg grating sensor." Opt. Commun., 192, 213-217( 2001)

[3] Ng, M.N.; Chen, Z.; Chiang, K.S. "Temperature compensation of long-period fiber grating for refractive-index sensing with bending effect.” IEEE Photon. Technol. Lett., 14, 361-367(2002)

[4] J.B. Judkins, J.R. Pedrassani, D.J. DiGiovanni, A.M. Vengsarkar, in Optical Fiber Communication Conference, Vol. 2, 1996 OSA Technical Digest Series (Optical Society of America, Washington, D.C., 1996), paper PD1.

[5] Shima, K.; Himeno, K.; Sakai, T.; Okude, S.; Wada, A.; Yamauchi, R., "A novel temperature-insensitive longperiod fiber grating using a boron-codoped-germanosilicate-core fiber," Optical Fiber Communication. OFC 97., Conference on , vol., no., pp.347,348, 16-21 Feb 1997

[6] Kwang Taek Kim, In Soo Kim, Cherl-Hee Lee, Jonghun Lee "A temperature-insensitive cladding-etched fiber bragg grating using a liquid mixture with a negative thermo-optic coefficient", Sensors, 12, 7886-7892(2012)

[7] K. Zhou, X. Chen, Y. Lai, K. Sugden, L. Zhang, and I. Bennion, "In-fiber polymer/glass hybrid waveguide Bragg grating," Opt. Lett. 33, 1650-1652 (2008).

[8] Zhiyi Zhang, Ping Zhao, Peng Lin, Fengguo Sun, "Thermo-optic coefficients of polymers for optical waveguide applications," Polymer, 47(14), 28 Pages 4893-4896(2006)

[9] A Chatak, [Optics],. Tata McGraw-Hill Education Chapter 29, 3 (2009) 